\title{
MIXED INTEGER SIMULATION OPTIMIZATION FOR PETROLEUM FIELD DEVELOPMENT UNDER GEOLOGICAL UNCERTAINTY
}

\author{
Honggang Wang \\ Industrial and Systems Engineering \\ Rutgers University \\ Piscataway, NJ 08854, USA
}

\begin{abstract}
Optimal development of oil and gas fields involves determining well locations in oil reservoirs and well control through the production time. Field development problems are mixed-integer optimization problems because the well locations are dened by integer-valued block indices in the discrete reservoir model, while the well control variables such as bottom hole pressures or injection rates are continuous. Reservoir simulation software is used to evaluate production performance given a well placement and control plan. In the presence of reservoir uncertainty, we sample and simulate multiple model realizations to estimate the expected eld performance. We present a retrospective optimization using dynamic simplex interpolation (RODSI) algorithm for oil field development under uncertainty. The numerical results show that the RODSI algorithm efficiently finds a solution yielding a $20 \%$ increase (compared to a solution suggested from heuristics) in the expected net present value (NPV) over 30 years of reservoir production for the considered Brugge case.
\end{abstract}

\section{INTRODUCTION}

Simulation optimization (SO) has gained increasing attention in the operations research and industry communities (Fu et al. 2005; Fu 2002; Pasupathy and Henderson 2011; Pichitlamken and Nelson 2003). Much research in simulation optimization has been focused on either continuous (Jin and Schmeiser 2003, Kushner and Yin 1997) or integer (Wang et al. 2012; Hong and Nelson 2006) optimization problems. Nonetheless, SO problems with both integer and continuous decision variables arise in various applications (Martin et al. 2006; Cameron and Durlofsky 2012). In design of supply chain systems (Glasserman and Wang 1998, Truong and Azadivar 2003), for example, the decision variables can be numbers of distribution centers, numbers of transportation vehicles, reorder points and quantities, service times and others. In oil and gas field development, well locations (integer valued within a discrete reservoir model) and well controls (e.g., bottom hole pressures or production rates are continuous decision variables) are often considered jointly (Cameron and Durlofsky 2012; Bellout et al. 2012).

Development of mixed integer simulation optimization (MISO) algorithms is difficult due to the mixture nature of the solution space and stochasticity of the objective function. In the case of oil field development, for example, it is not uncommon that a reservoir simulator uses hours of computing time to compute the field performance for one solution. To account for geological uncertainty, hundreds or more model realizations could be considered for each solution evaluation and the optimization method could evaluate hundreds to thousands of solutions dependent on the methods employed. In addition, petroleum field development usually involves both integer-valued variables (e.g., well locations are integer labeled drilling blocks as the field has been discretized to reservoir blocks) and continuous variables (e.g., bottom hole pressures). Finding the optimal development plan including well locations and control for oil fields can significantly increase production revenues for energy companies. Thus practically robust and efficient solution methods for MISO problems are much desired in petroleum industry. 
MISO is a less studied (Hemker et al. 2008; Keskin et al. 2010) subject in operations research compared to continuous or discrete simulation optimizations. We propose a new retrospective optimization using dynamic simplex interpolation (RODSI, described in Section 2) that can be applied to many real-world MISO applications in various areas such as environmental sciences (e.g., $\mathrm{CO}_{2}$ sequestration, subsurface water resources), financial engineering, and healthcare systems (e.g., service scheduling, treatment planning, facilities and operations).

\subsection{Mixed-Integer Simulation Optimization Problem Statement}

We consider mixed-integer simulation optimization (MISO) problems where the objective function and some constraint functions are implicitly defined by stochastic simulation. A formal statement of MISO problems considered in this work is provided as follows.

$$
\begin{array}{ll}
\text { (P) } \max & g(x)=\lim _{m \rightarrow \infty} \hat{g}(x, m), \quad x \in \mathbb{Z}^{d_{1}} \times \mathbb{R}^{d_{2}}, \\
\text { Subject to } & c_{i}(x) \leq 0, i=1,2, \ldots, l, \\
& h_{j}(x) \leq 0, j=1,2, \ldots, n .
\end{array}
$$

Let $\mathbb{X}$ denote the feasible region in $(\mathrm{P})$ and $d=d_{1}+d_{2}$. Here the feasible region $\mathbb{X}$ is a subset in the $d$-dimensional mixed space specified by a number of deterministic constraints $c_{i}(x) \leq 0, i=1,2, \ldots, l$ and stochastic constraints $h_{j}(x) \leq 0, j=1,2, \ldots, n$. The objective function $g$ and stochastic constraints $h_{i}(x)$ can only be observed through simulation processes $G$ and $H$ respectively. Specifically, $G$ and $H$ provide consistent estimates $\hat{g}$ and $\hat{h}_{j}$ for $g$ and $h_{j}$ respectively; that is, $\lim _{m \rightarrow \infty} \hat{g}(x, m)=g(x)$ with probability 1 (w.p.1) and $\lim _{m \rightarrow \infty} \hat{h}_{j}(x, m)=h_{j}(x)$ w.p.1, for every $x \in \mathbb{X}, j=1,2, \ldots, n$, where $m$ denotes some measure of computational effort that often is the sample size of computer simulations and $\hat{g}(x, m)=\frac{1}{m} \sum_{k=1}^{m} G\left(x, \omega_{k}\right)$. The stochastic parameters $\omega$ are sampled from the parameter space $\Omega$ associated with the MISO problem. The goal of $(\mathrm{P})$ is to seek a maximizer $x^{*}$ of the limiting function $g$ in $\mathbb{X}$.

\subsection{Local Optimality for MISO}

In this paper, we develop new MISO methods for identifying a local maximum of $(\mathrm{P})$, given the following neighborhood definition. Let $x=\left(x_{1}, x_{2}\right), x_{1} \in \mathbb{Z}^{d_{1}}, x_{2} \in \mathbb{R}^{d_{2}}$ be a feasible solution in $\mathbb{X}$. The $\delta$-neighborhood $N_{\delta}(x)$ of $x$ includes all the points $y=\left(y_{1}, y_{2}\right) \in \mathbb{X}$ so that $\left\|x_{1}-y_{1}\right\| \leq 1,\left\|x_{2}-y_{2}\right\| \leq \delta$, where $y_{1} \in \mathbb{Z}^{d_{1}}$, $y_{2} \in \mathbb{R}^{d_{2}}$ and $\|\cdot\|$ represents the Euclidean distance. A feasible solution $x^{*}$ is a local maximum of (P) if there exists a $\delta>0$ such that $g\left(x^{*}\right) \geq g(y)$ for any $y \in N_{\delta}\left(x^{*}\right)$.

We develop RODSI to efficiently solve MISO problems of (P) for local optima. RODSI generates a sequence of sample-path problems $P_{k}$ with objective functions $\hat{g}\left(x, m_{k}\right)$ and increasing sample sizes $m_{k}$. RODSI solves $P_{k}$ sequentially via a local search based on DSI for a sequence of approximate local optima $X_{k}$. Some discussion on general RO algorithm performance and convergence properties can be found in (Wang et al. 2013; Wang and Schmeiser 2008; Wang 2012).

In this work, we focus on the RODSI design and numerical demonstration of RODSI using a petroleum field development example. Specifically, we will focus on efficient sampling methods for generating representative RO sample-path problems $P_{k}$ and effective strategy for scaling issues between integer and continuous variables.

\section{RODSI ALGORITHM DESIGN}

A generic RODSI framework is described in (Wang 2012), where some convexity assumptions on the objective function were made for global convergence. In this paper, we consider MISO problems with nonconvex objective functions of $g$. 


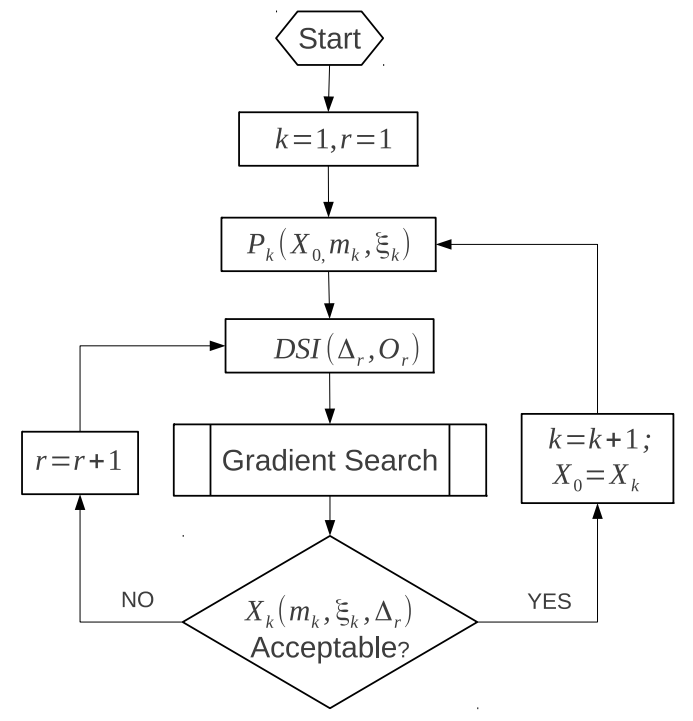

Figure 1: Flowchart of RODSI.

\subsection{Sampling Parameters}

RODSI uses a conceptually simple framework - during the $k$ th retrospective iteration, the DSI based local search attempts to identify an approximate local maximal solution $X_{k}$ of the sample-path function $\hat{g}\left(x, m_{k}\right)$. RODSI considers the following sample-path problems $P_{k}$ iteratively.

$$
\left(P_{k}\right): \quad \text { Find } X_{k} \in \mathbb{X} \text { such that } \hat{g}\left(x, m_{k}\right) \leq \hat{g}\left(X_{k}, m_{k}\right), \quad \forall x \in N_{\delta}\left(X_{k}\right) \text {, for some } \delta>0 \text {. }
$$

Where the feasible region $\mathbb{X}$ is defined by both deterministic constraints $c_{i}(x) \leq 0, i=1,2, \ldots, l$, stochastic constraints $\hat{h}_{j}\left(x, m_{k}\right) \leq 0, j=1,2, \ldots, n$, and the mixed-integer space $\left\{\mathbb{Z}^{d_{1}} \times \mathbb{R}^{d_{2}}\right\}$. As can be seen from (1), the problem $P_{k}$ involves generating a sample-path function using the sample $\xi_{k}=\left\{\omega_{1}, \omega_{2}, \ldots, \omega_{m_{k}}\right\}$ of sample size $m_{k}$. Effective means to determine $m_{k}$ and $\xi_{k}$ are critical to RODSI performance.

While RODSI has a RO framework similar to other retrospective algorithms (Jin and Schmeiser 2003; Chen and Schmeiser 2001; Wang et al. 2012), RODSI differs from previous retrospective algorithms in that RODSI considers integer and continuous variables jointly and uses a new local search based on the stochastic dynamic simplex interpolation.

Figure 1 shows the flowchart for a generic RODSI design. This algorithm has three major components: (i) construction of sample-path problems $P_{k}$, (ii) application of DSI with a selected simplex size $\Delta_{r}$ and simplex orientation $O_{r}$, and (iii) solving $P_{k}$ using a local search method via DSI. The construction of $P_{k}$ involves determining the important algorithm parameters including the sample size $m_{k}$ and the sample of $\xi_{k}$. We propose a hierarchical sampling theme that statistically determines sample sizes $m_{k}$ and representative samples $\xi_{k}$ based on the properties of the considered stochastic parameters. Detailed discussion of stochastic hierarchical sampling and its application for the Brugge case will be provided in Section 4.

Note that the RODSI logic in Figure 1 does not have a stopping rule in place. In practice, however, RODSI must terminate after a finite number of optimization iterations. The possible stopping rules could be specified by the limit of computing budget, a maximum number $k$ of sample-path problems solved, or finding a solution that can not be improved by subsequent sample-path problems with large enough sample sizes. 


\subsection{DSI Based Local Search}

Recent work (Wang 2012) extends simplex linear interpolation (SLI) to SLI with varying dimensions for mixed-integer optimization problems. In this work, we design a new dynamic simplex interpolation (DSI) method for MISO problems. DSI differs from Wang (2012) in that DSI allows stochastic change of the orientations of simplex. RODSI change simplex orientations across iterations to provide local optimum convergence.

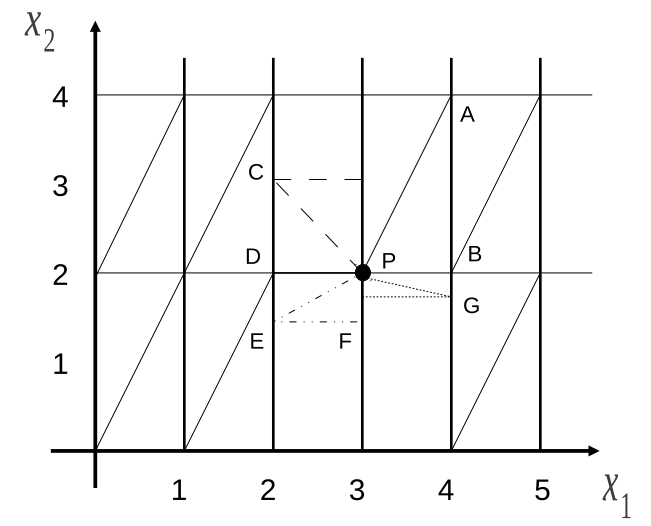

(a) Dynamic simplex linear interpolation.

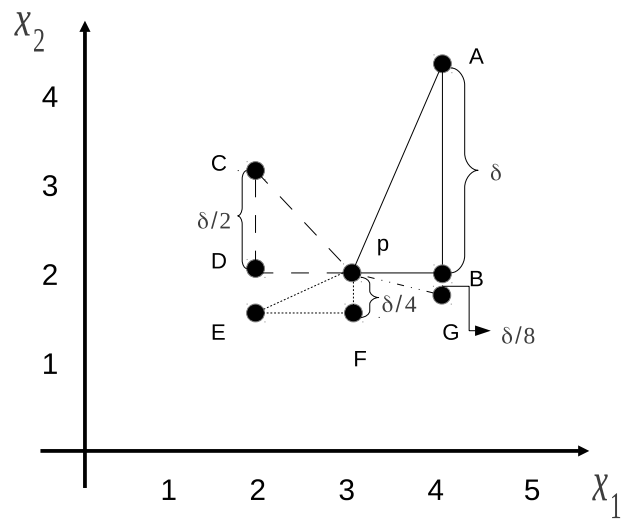

(b) Dynamic simplex interpolation at a local optimum.

Figure 2: Dynamic simplex interpolation on a mixed solution space.

To illustrate the basic ideas of DSI, we look at a 2-dimensional example shown in Figure 2. Suppose that we consider an MISO problems with one integer-valued variable $x_{1}$ and one continuous variable $x_{2}$ and they are both positive. The objective function is assumed to be evaluated exactly. As shown in Figure 2 (a), the feasible region $\mathbb{X}$ for this case contains an infinite number of rays in the positive quadrant of $\mathbb{R}^{2}$. We first apply SLI in $\mathbb{X}$ with the simplex size $\Delta_{1}=(1,2)$ (i.e. the positive quadrant of $\mathbb{R}_{+}^{2}$ is partitioned into triangles of dimensions 1 and 2 for $x_{1}$-axis and $x_{2}$-axis respectively). The function value at any point $y \in \mathbb{R}_{+}^{2} \backslash \mathbb{X}$ in the $\operatorname{SLI}\left(\Delta_{1}\right)$ constructed space is the convex combination of the function values at the vertices of the corresponding triangle that contains the point $y$. This continuous surface constructed by $\operatorname{SLI}\left(\Delta_{1}\right)$ allows us to use a gradient-based (or more precisely subgradient based for grid points (Clarke 1990)) local search to quickly find a local optimum; let us assume that such a continuous search procedure always returns a local optimum that is a grid point of $\operatorname{SLI}\left(\Delta_{1}\right)$. In Figure 2(b), suppose we obtain a candidate solution $p$ after the first gradient continuous search via $\operatorname{SLI}\left(\Delta_{1}\right)$. The grid looks quite coarse and a finer SLI will likely locate a better solution near the current candidate solution $\mathrm{p}$. We apply a second SLI with the simplex size $\Delta_{2}=(1,1)$, the $\operatorname{SLI}\left(\Delta_{2}\right)$ based continuous search checks solutions within a smaller simplex (triangle) with vertices (p,C,D), but no better solution found. Note that the second SLI is used to generate smaller simplex with a different orientation within a local rectangle of size $\Delta_{1}$. Such a change of simplex orientation allows the search routine to evaluate solutions located in different directions to the current candidate $\mathrm{p}$. For this example case, we apply SLI four times with different simplex sizes $\Delta$ and orientations $O$ and find that $\mathrm{p}$ is the best solution among the visited 8 solutions (p,A,B,C,D,E,F,G). If we apply a sequence of SLI with simplex sizes $\Delta$ (for those corresponding to continuous variable) asymptotically decreasing to 0 , we expect that the final solution returned is a local optimum to the original MISO problem (P). Note that we only change the simplex sizes corresponding to the continuous variables. For integer-valued variables, the the corresponding simplex dimensions remain one. This guarantees that the grid points generated by any $\operatorname{SLI}(\Delta)$ are always feasible in $\mathbb{X}$.

The above example shows how to apply a number of dynamic SLI to find an approximate local optimum quickly. DSI is computationally efficient because late SLI is only applied locally in real time and is only performed when the search algorithm requests. We notice that DSI may generate non-continuous surfaces 
with varying simplex sizes and orientations, but for a local optimum search, it will not be a problem for most cases.

The proposed DSI based continuous search is embedded (as the RODSI's core optimizer) in the retrospective optimization framework for solving sample-path problems $P_{k}$.

\section{BRUGGE OIL FIELD DEVELOPMENT}

The Brugge oil field (Peters et al. 2010) is a synthetic 3-dimensional model constructed based on a North Sea reservoir that has been widely used as example case in petroleum and energy resources engineering. The 3 -dimensional discretized reservoir model consists of $139 \times 48 \times 9$ blocks and each block is of dimensions $100 \times 100 \times 6$ meters. The field has 10 fixed injection wells with full completion (from the top block through the bottom block of the reservoir). These 10 injection wells inject high-pressure water down to the reservoir and push the oil and water out from the production wells. In development of the Brugge oil field (Wang et al. 2012; Wang et al. 2010; Peters et al. 2010), we need to determine the drilling locations and operation control of 11 new vertical production wells. The objective for this field development project is to find such optimal well locations and control so that the net present value (NPV) of the field production through the period of 30 years will be maximized.

We now discuss the stochastic nature of the problem. In the general case, many aspects of the reservoir model are uncertain (Mattax and Dalton 1990; Caers et al. 2010). Major uncertainties include the type of geological depositional system and associated parameters, the presence and location of large-scale faults and fractures, and the spatial correlation structure of rock properties such as porosity and permeability. The parameters quantifying the efficiency of the displacement of oil by the injected water (e.g., residual oil saturation, endpoint relative permeabilities), as well as the depth of the oil-water contact, can also be uncertain. In an attempt to account for these and other uncertainties, multiple realizations of the reservoir model are constructed. Depending on the number and ranges of the parameters considered, there can be tens, hundreds or thousands of model realizations. Predicted reservoir performance is then reported in terms of expected values and cumulative distribution functions for essential quantities such as oil production rate as a function of time.

For the Brugge case, we consider four major uncertain parameters: permeability, facies (presence of faults), fluvial property (channels), and porosity. The key property that impacts fluid flow is the rock permeability (essentially a conductivity measure relating flow rate to pressure gradient), which can vary by many orders of magnitude over the field (Figure 3). Facies and fluvial properties are categorical with 2 and 3 possible values respectively for this case. All of these four reservoir parameters are highly uncertain due to the complex geological structure of the Brugge field and the limited data available. To sufficiently capture the reservoir uncertainty for the Brugge field, 104 reservoir realizations were generated by sampling data from these considered geological parameters. Figure 4 shows five model realizations of the first layer of the Brugge reservoir. As for many realistic reservoirs, the geological properties of the Brugge field (e.g., the permeability field shown in Figure 3) are highly heterogeneous over the reservoir and the optimal location of wells is not obvious to determine.

We consider drilling 11 new production wells in the Brugge field. For each production well, two decision variables $(I, J)$ are considered for this optimization problem, where $(I, J)$ represent the integer coordinates for the drilling block for the well. All of the 11 production wells are fully completed, i.e. the wells are perforated from a top block at layer 1 through a bottom block at layer 9 . For any production well $l$, the location variables $\left(I_{l}, J_{l}\right)$ must be within the field region $\{[1,139] \times[1,48]\} \cap \mathbb{Z}^{2}$. We also consider the well-distance constraints $\left\|\left(I_{l}, J_{l}\right)-\left(I_{j}, J_{j}\right)\right\| \geq 5$ between well $l$ and well $j$, i.e. the wells must be at least 5 blocks away from each other within the Brugge field. We also consider stochastic constraints defined by the permeability values. The blocks with significant low permeability (e.g., lower than $10^{-5}$ millidarcy) will be considered infeasible location to drill a well.

We also consider continuous variables of well control in the Brugge case. Specifically the bottom hole pressures of the production wells are considered to be decision variables, which vary every 10 years for 

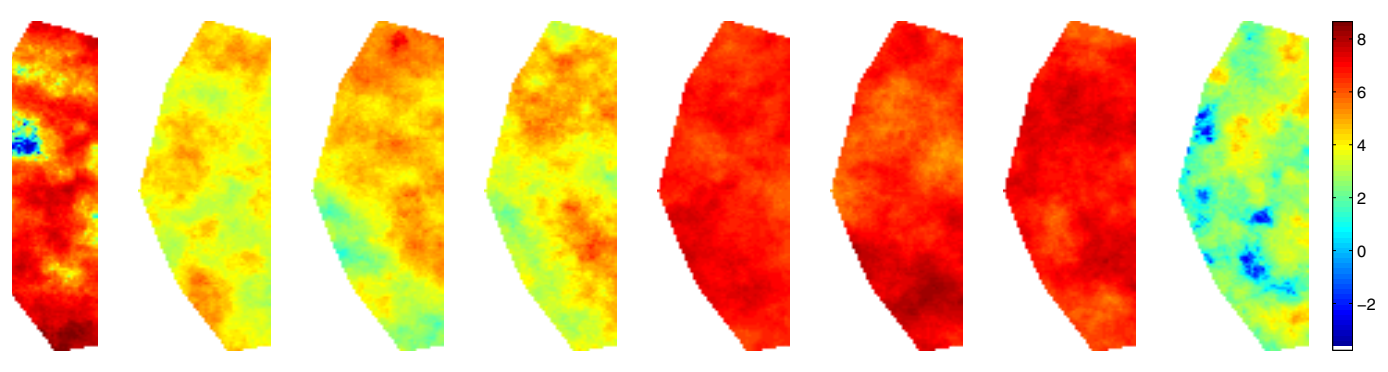

(a) Permeability field for nine layers of Brugge realization 1.

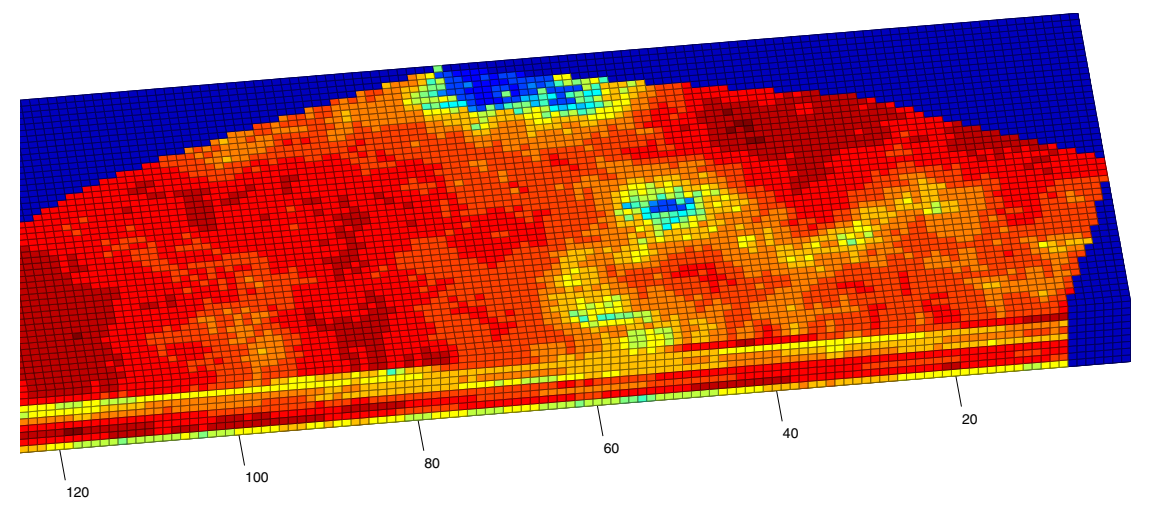

(b) 3D model of Brugge permeability.

Figure 3: Brugge 3-dimensional permeability field of realization 1.

each production well. Suppose that for production well 1 , the control variables $\left(y_{1}, y_{2}, y_{3}\right)$ represent the bottom hole pressures for the production periods of year 1 to year 10, year 11 to year 20, and year 21 to year 30, respectively for the three 10-year intervals. For this case, the bottom hole pressures for the 10 injection wells are fixed to be a constant of 2611 psi. With this setting, we have a total of 55 continuous variables. The control variables for the bottom hole pressures of production wells are constrained to the interval $[300,1800]$ psi.

Thus the total number of optimization variables for this problem is 55 including 22 integer-valued well location variables and 33 well control variables. We formulate the constrained MISO problem associated with the optimal development of the Brugge oil field as follows.

$$
\max _{x \in \mathbb{X}} g=\frac{1}{104} \sum_{i=1}^{104} G\left(x, \omega_{i}\right)=\frac{1}{104} \sum_{i=1}^{104}\left[\sum_{j=1}^{T} \frac{p_{o} q_{i, j}^{o}-p_{w_{1}} q_{i, j}^{w_{1}}-p_{w_{2}} q_{i, j}^{w_{2}}}{(1+\lambda)^{j}}\right],
$$

where $p_{o}$ is the oil price ( $\$ 80 / \mathrm{bbl}, 1$ barrel is equal to 42 gallons), $p_{w_{1}}$ and $p_{w_{2}}$ are the water production and injection costs (both taken to be $\$ 5 / \mathrm{bbl}$ ), $\lambda=0.0234$ is the discount rate per unit time, $q_{i, j}^{o}, q_{i, j}^{w_{1}}$ and $q_{i, j}^{w_{2}}$ are the oil and water production rates and the water injection rate, respectively, for the $i^{t h}$ model realization and the $j^{\text {th }}$ time period. Water production is a cost because equipment and energy are required to produce, separate, and dispose of water. We use the ECLIPSE ${ }^{\mathrm{TM}}$ reservoir simulator in this study. One call of $G$ gives one observation of production quantities of $q_{i, j}^{o}, q_{i, j}^{w_{1}}, q_{i, j}^{w_{2}}, j=1,2, \ldots, T$, given an $x$ and the $i^{\text {th }}$ realization of model $\omega_{i}$, which in turn provides one observation of $g$. It takes about 5 minutes to complete a simulation run of $G$ for a single realization of the Brugge model, running on a Windows PC with a $2.66 \mathrm{Ghz} \mathrm{CPU}$ and $1.95 \mathrm{G}$ of RAM. For this case, we use a cluster with 20 nodes for faster optimization runs. 


\section{NUMERICAL EXPERIMENTS}

We discuss some implementation detail of RODSI and present the numerical results of applying RODSI to the optimal development of Brugge field.

\subsection{RODSI Implementation}

We determine RODSI parameters as follows. The sample sizes $m_{k}$ and sample $\xi_{k}$ have been selected based on the hierarchical approach described below. The simplex sizes $\Delta_{r}$ are reduced by a half iteratively and the orientations $O_{r}$ are randomly selected. The nonlinear optimizer in RODSI is a DSI based local search.

\subsubsection{Sampling Parameters}

For the Brugge case, with some simple statistical analysis, the most important reservoir parameter is permeability followed by facies and fluvial properties; the rock porosity turns out least important. The permeability field was generated based on three types of constructions (Peters et al. 2010): deterministically with a porosity/permeability regression (KS), deterministically with a porosity/permeability regression per facies (KM, facies must be enabled for KM) and stochastically using co-Kriging on porosity (KP). Therefore we can sample permeability realizations from these three major groups. The facies classify reservoir models into two groups: enabled (FY) versus not enabled (FN). The fluvial property, if facies enabled (FY), has two possible values for this case, labeled with SF and SS respectively. Note that there are correlations among these three parameters, both permeability and fluvial are dependent on the facies property. Therefore, for this case, the population of model realizations can be classified into three groups based on permeability construction, five groups based on the combination of permeability and facies, and eight groups considering the permeability, facies, and fluvial properties.
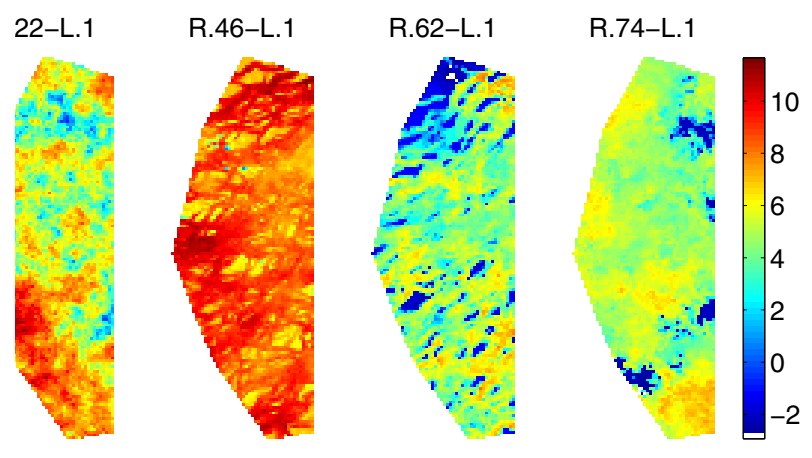

Figure 4: Permeability variations among five stochastic realizations of the Brugge field (first layer).

We consider a hierarchical sampling approach based on the three most important parameters. For the first sample-path problem $P_{1}$ we sample realizations based on permeability construction. Because the permeability field can be sampled based on three construction techniques, it is natural to generate three realizations with each from one construction of KS, KM or KP. We merge two groups of KS and KM as they are similar in terms of production performance; so we consider two major groups of KS/KM and KP. For $P_{1}$, thus we let $m_{1}=2$ and $\xi=\left\{\omega_{1}, \omega_{2}\right\}$. With a simple simulation verification, we see two randomly selected realizations from KS/KM and KP respectively are quite representative of the population of 104 realizations (generated by reservoir engineers to cover most parameter uncertainties) in terms of field production performance. For a more-accurate estimation of $g$, within $P_{2}$, we sample more realizations based on two parameters: permeability and facies. Thus, for $P_{2}$ we set $m_{2}=5$ and the sample $\xi_{2}$ consists of five realizations from the jointly-sampled realizations, i.e. one from KS-FY, KS-FN, KM-FY, KP-FY, and KP-FN 

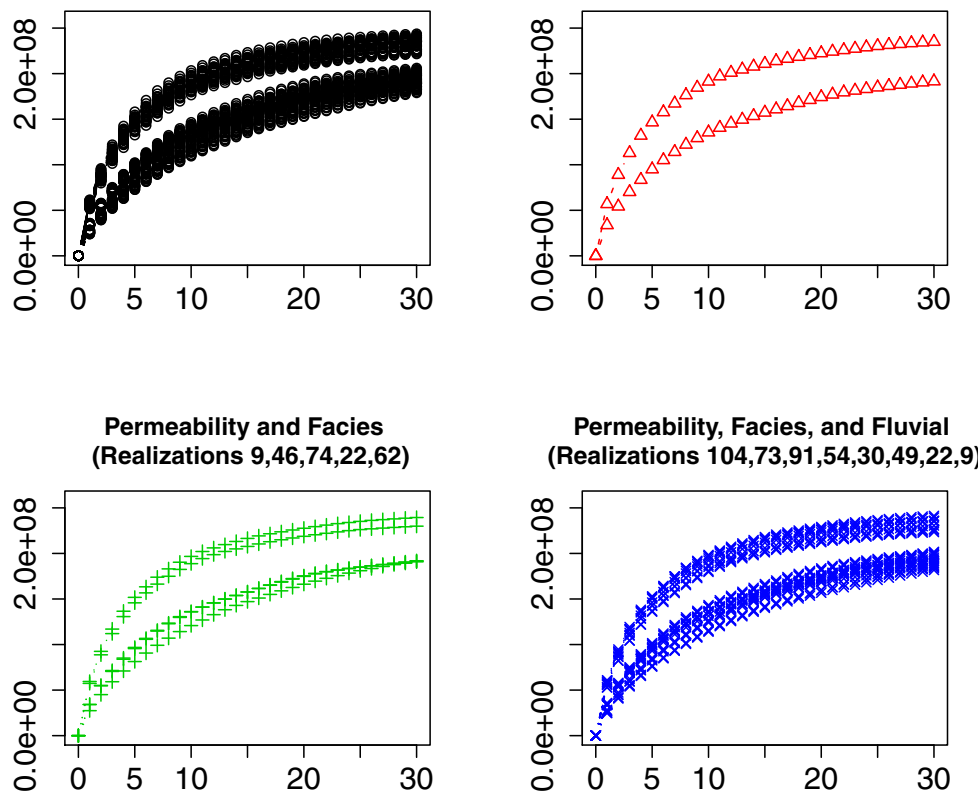

Figure 5: Simulation results for hierarchical subsets of realizations using initial well locations. Cumulative oil recovered versus time in years is plotted.

respectively. For $P_{3}$, we consider the three most important parameters of permeability, facies, and fluvial properties. Therefore we set $m_{3}=8$ and $\xi_{3}$ selects one realization from each of 8 combinations of these three parameters. Figure 5 shows that the cumulative productions for sampled subsets of realizations based on such hierarchical sampling. The production profiles of the selected subsets of realizations approximate those of the population realizations well and better when the sample sizes get larger. For better accuracy and possible better solutions, RODSI generates and solves the last $P_{4}$ with 32 realizations including three realizations from each of the eight groups of realizations.

\subsubsection{Scaled Gradient Search for MISO}

For many MISO problems associated with oil field development problems, the derivatives of objective function corresponding to the integer valued versus continuous variables can have orders of magnitude difference. For this Brugge case, the derivatives of integer variables are in the order of $10^{8}$ whereas those of continuous variables are in the order of $10^{4}$. This scaling issue is significant so that a usual gradient search will not be effective for the search in the control variable space because the search in the integer subspace is dominating. To overcome this challenge, we scale the derivatives for integer and continuous variables accordingly, that is, scaling up the continuous variable derivatives four orders of magnitude. With such a treatment, the scaled gradient search will proceed within both steep integer variable subspace and flat continuous variable subspace.

\subsection{Numerical Results}

We apply the RODSI algorithm described above to the Brugge case. The initial solution was given based on engineering heuristics. Figure 6 shows the optimization progress through one run of RODSI. The performance curve in Figure 6(a) reports the current maximal average NPV versus the number of simulation calls performed. This run of RODSI solves four sample-path problems and thus RODSI returns four RO iterate solutions. Together with the initial solution, we evaluate these five solutions with the total of 104 realizations. The true improvement in NPV from the RODSI returned solutions for this 
Brugge field production is plotted in the Figure $6(\mathrm{~b})$. The results suggest that RODSI returns the final solution with about 1.85 billion dollars increase of average NPV. We also notice that the sampling errors $\delta_{k}=\left|\hat{g}\left(X_{k}, m_{k}\right)-g\left(X_{k}\right)\right|, k=0,1,2,3,4$ (the gap between the approximation and the true evaluation of average NPV) reduce progressively as the sample sizes $m_{k}$ increase.

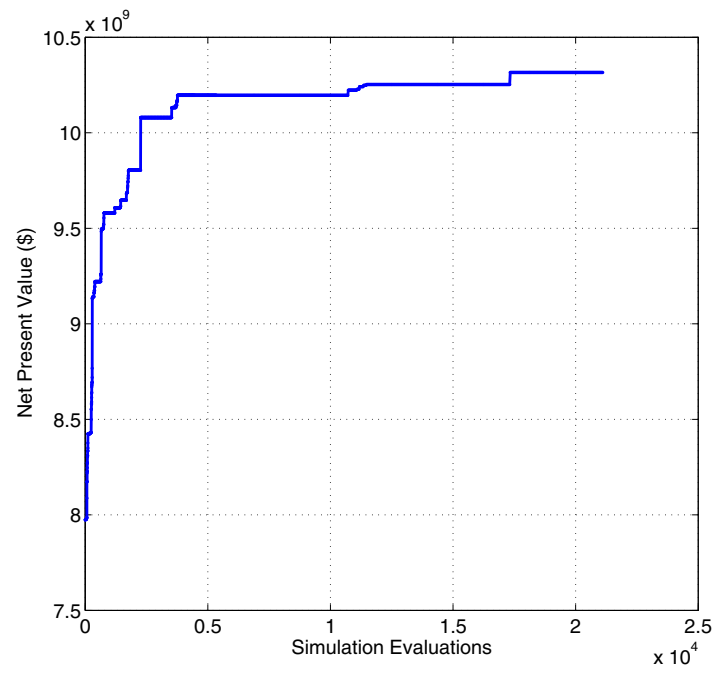

(a) RODSI optimization progress for Brugge case.

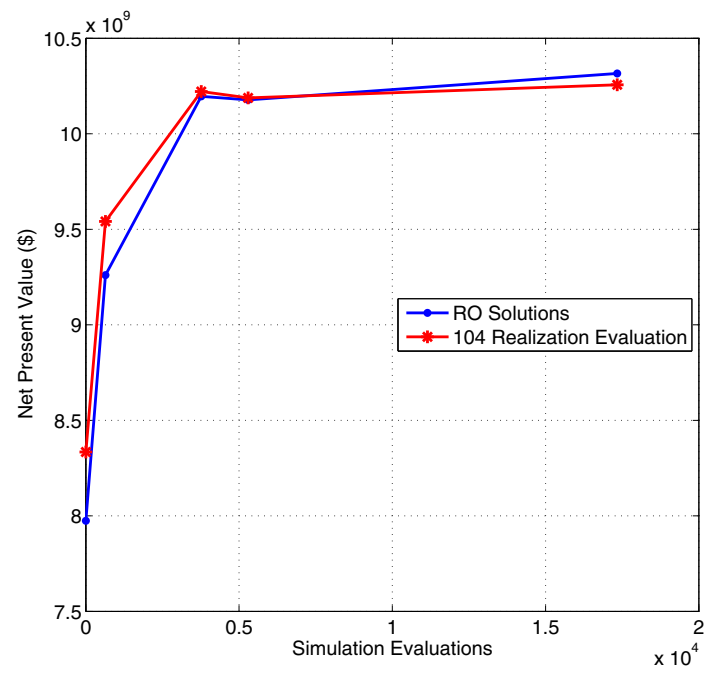

(b) RODSI optimization progress verification.

Figure 6: RODSI performance on Brugge field development.

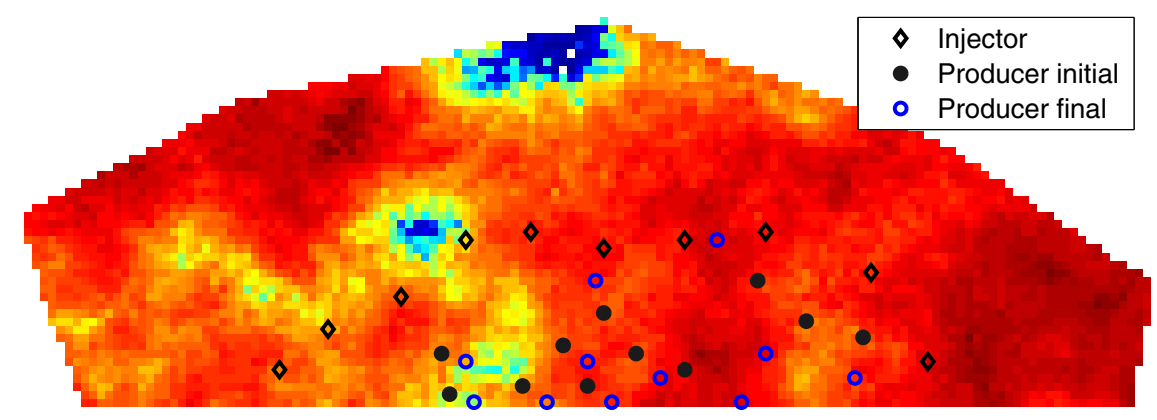

Figure 7: Optimized well placement for Brugge field.

Figure 7 shows the initial well locations and RODSI optimized well locations. The optimization procedure moves the wells toward the reservoir boundary to obtain higher NPV. The initial well controls of bottom hole pressure are set to be the same 750 psi through three 10-year intervals for all the 11 production wells. The optimized control values of 11 production wells from year 1 to year 10 are $[762,763,762,779,766,767,763,757,766,769,767]$, from year 11 to year $20[763,766,767,766,762,767$, $767,763,767,763,783]$, and the last 10 years from year 21 to year $30[767,763,769,766,766,782,763,763$, $768,763,767]$.

\section{CONCLUDING REMARKS}

In this work we implemented a retrospective optimization using dynamic simplex interpolation (RODSI) algorithm for mixed-integer simulation optimization problems. The promising results for our target application - optimizing the placement of oil wells and well operations in a medium-sized realistic model under 
Wang

uncertainty - suggest that RODSI can be used for this class of problems to provide significant improvement in net present value. Future work will include algorithm convergence analysis and performance comparisons with other methods.

\section{ACKNOWLEDGMENTS}

We thank Dr. David Echeverri from T.J. Watson IBM for valuable comments and suggestions on this work. Cluster computing for reservoir simulation and optimization has been provided by the high-performance computing center at Norwegian University of Science and Technology (NTNU).

\section{REFERENCES}

Bellout, M. C., D. E. Ciaurri, L. J. Durlofsky, B. Foss, and J. Klep. 2012. "Joint Optimization of Oil Well Placement and Controls". Computational Geosciences 16 (4): 1061-1079.

Caers, J., C. Scheidt, and K. Park. 2010. Modeling Uncertainty of Complex Earth Systems in Metric Space. Springer-Verlag, Berlin-Heidelberg.

Cameron, D. A., and L. J. Durlofsky. 2012. "Optimization of Well Placement, $\mathrm{CO}_{2}$ Injection Rates, and Brine Cycling for Geological Carbon Sequestration”. International Journal of Greenhouse Gas Control 10:100-112.

Chen, H., and B. W. Schmeiser. 2001. "Stochastic Root Finding Via Retrospective Approximation". IIE Transactions 33:259-275.

Clarke, F. H. 1990. Optimization and Nonsmooth Analysis. Society for Industrial and Applied Mathematics, Philadelphia.

Fu, M. C. 2002. "Optimization for Simulation: Theory vs. Practice (Feature Article)". INFORMS Journal on Computing 14 (3): 192-215.

Fu, M. C., F. W. Glover, and J. April. 2005. "Simulation Optimization: a Review, New Developments, and Applications". In Proceedings of the 2005 Winter Simulation Conference, edited by M. E. Kuhl, N. M. Steiger, F. B. Armstrong, and J. A. Joines, 83-95. Orlando, FL, USA: Institute of Electrical and Electronics Engineers, Inc.

Glasserman, P., and Y. Wang. 1998. "Leadtime-Inventory Trade-offs in Assemble-to-Order Systems". Operations Research 46:858-871.

Hemker, T., K. R. Fowler, M. W. Farthing, and O. V. Stryk. 2008. "A Mixed-Integer Simulation-Based Optimization Approach with Surrogate Functions in Water Resources Management". Optim Eng 9:341360.

Hong, J. L., and B. L. Nelson. 2006. "Discrete Optimization via Simulation Using COMPASS". Operations Research 54 (1): 115-129.

Jin, J., and B. W. Schmeiser. 2003. "Simulation-Based Retrospective Optimization of Stochastic Systems". In Proceedings of the 2003 Winter Simulation Conference, edited by S. Chick, P. J. Sánchez, D. Ferrin, and D. J. Morrice, 543-547. Piscataway, New Jersey: Institute of Electrical and Electronics Engineers, Inc.

Keskin, B. B., S. H. Melouk, and I. L. Meyer. 2010. "A Simulation-Optimization Approach for Integrated Sourcing and Inventory Decisions". Computers and Operations Research 37 (9): 1648-1661.

Kushner, H. J., and G. G. Yin. 1997. Stochastic Approximation Algorithms and Applications. Springer-Verlag, New York.

Martin, A., M. Möller, and S. Moritz. 2006. "Mixed Integer Models for the Stationary Case of Gas Network Optimization". Mathematical Programming 105:563-582.

Mattax, C. C., and R. L. Dalton. 1990. Reservoir Simulation. SPE Monograph Volume 13.

Pasupathy, R., and S. Henderson. 2011. "SimOpt: a Testbed of Simulation-Optimization Problems (Invited Paper)". In Proceedings of the 2011 Winter Simulation Conference, edited by S. Jain, R. R. Creasey, 
J. Himmelspach, K. P. White, and M. Fu, 412-421. Piscataway, New Jersey: Institute of Electrical and Electronics Engineers, Inc.

Peters, L., R. J. Arts, G. K. Brouwer, C. R. Geel, S. Cullick, R. J. Lorentzen, Y. Chen, K. N. B. Dunlop, F. C. Vossepoel, R. Xu, P. Sarma, A. H. Alhuthali, and A. C. Reynolds. 2010. "Results of the Brugge Benchmark Study for Flooding Optimization and History Matching". SPE Reservoir Evaluation \& Engineering 13 (3): 391-405.

Pichitlamken, J., and B. L. Nelson. 2003. "A Combined Procedure for Optimization via Simulation". ACM Trans. Modeling and Comput. Simulation 13:155-179.

Truong, T. H., and F. Azadivar. 2003. "Simulation Optimization in Manufacturing Analysis: Simulation Based Optimization for Supply Chain Configuration Design". In Proceedings of the 2003 Winter Simulation Conference, edited by S. Chick, P. J. Sánchez, D. Ferrin, and D. J. Morrice, 1268-1275. Piscataway, New Jersey: Institute of Electrical and Electronics Engineers, Inc.

Wang, H. G. 2012. "Retrospective Optimization of Mixed Stochastic Systems Using Dynamic Simplex Interpolation”. European Journal of Operational Research 217 (1): 141-148.

Wang, H. G., D. E. Ciaurri, and L. J. Durlofsky. 2010. "Use of Retrospective Optimization for Placement of Oil Wells under Uncertainty". In Proceedings of the 2010 Winter Simulation Conference, edited by B. Johansson, S. Jain, J. Montoya-Torres, J. Hugan, and E. Yücesan, 1750-1757. Piscataway, New Jersey: Institute of Electrical and Electronics Engineers, Inc.

Wang, H. G., D. Echeverria, and L. J. Durlofsky. 2012. "Optimal Well Placement under Uncertainty Using a Retrospective Optimization Framework". Society of Petroleum Engineers Journal 17 (1): 112-121.

Wang, H. G., R. Pasupathy, and B. W. Schmeiser. 2013. "Integer-ordered Simulation Optimization Using R-SPLINE: Retrospective Search via Piecewise-Linear Interpolation and Neighborhood Enumeration”. ACM Transactions on Modeling and Computer Simulation:in press.

Wang, H. G., and B. W. Schmeiser. 2008. "Discrete Retrospective Optimization Using Linear Interpolation". In Proceedings of the 2008 Winter Simulation Conference, edited by S. J. Mason, R. R. Hill, L. Mönch, O. Rose, T. Jefferson, and J. W. Fowler, 502-508. Piscataway, New Jersey: Institute of Electrical and Electronics Engineers, Inc.

\section{AUTHOR BIOGRAPHY}

HONGGANG WANG is an assistant professor in the Department of Industrial and Systems Engineering at Rutgers University. His research interests lie in stochastic optimization, simulation modeling and analysis, and their applications in energy systems. His email address is honggang.w@rutgers.edu. 